\title{
Quantification of caval flow contribution to the lungs in vivo after total cavopulmonary connection with 4-dimensional flow magnetic resonance imaging
}

\author{
Pablo Bächler, $\mathrm{MD},{ }^{\mathrm{a}, \mathrm{b}}$ Israel Valverde, $\mathrm{MD},{ }^{\mathrm{c}}$ and Sergio Uribe, $\mathrm{PhD}^{\mathrm{a}, \mathrm{d}}$
}

Quantification of caval flow distribution to the right pulmonary artery (RPA) and left pulmonary artery (LPA) after total cavopulmonary connection (TCPC) is of great interest to understand the flow dynamics and energy loss in nonefficient cavopulmonary anastomosis, which may lead to failing Fontan circulation. In addition, uneven distribution of hepatic factors between the 2 lungs may explain the development of pulmonary arteriovenous malformations (PAVMs), leading to decreased systemic oxygenation. ${ }^{1,2}$

From the Biomedical Imaging Center ${ }^{\mathrm{a}}$ and School of Medicine, ${ }^{\mathrm{b}}$ Pontificia Universidad Católica de Chile, Santiago, Chile; Division of Imaging Sciences, ${ }^{\mathrm{c}} \mathrm{King}$ 's College London, NIHR Biomedical Research Centre at Guy's and St Thomas' NHS Foundation Trust, London, United Kingdom; and Radiology Department, ${ }^{\mathrm{d}}$ School of Medicine, Pontificia Universidad Católica de Chile, Santiago, Chile. Funding: Fondecyt 11100427, ACT 079, Vicerrectoria de Investigación, PUC. Disclosures: Authors have nothing to disclose with regard to commercial support. Received for publication Oct 28, 2011; accepted for publication Nov 7, 2011; available ahead of print Dec 12, 2011.

Address for reprints: Sergio Uribe, PhD, Radiology Department, Pontificia Universidad Católica de Chile, Marcoleta 367, Second Floor, Radiology Department, Hospital Clínico Universidad Catolica (E-mail: suribe@med.puc.cl).

J Thorac Cardiovasc Surg 2012;143:742-3

$0022-5223 / \$ 36.00$

Copyright (c) 2012 by The American Association for Thoracic Surgery doi:10.1016/j.jtcvs.2011.11.003
Dasi and colleagues ${ }^{2}$ recently reported the influence of the TCPC geometry in the inferior vena cava (IVC) flow distribution based on computational fluid dynamic simulations. They identified an ideal TCPC varying the offset between the IVC and the superior vena cava (SVC) and cardiac output split. ${ }^{2}$

In vivo evaluation of caval flow distribution after TCPC is difficult to achieve. Current quantification blood flow methods cannot estimate flow contributions from a particular vessel when more than 1 source of flow is present. Fogel and colleagues ${ }^{3}$ proposed to evaluate caval contribution to flow in the RPA and LPA using a spin labeling technique. Nevertheless, this method is not applicable to all patients undergoing the Fontan procedure, especially those with turbulent flow in the pulmonary arteries.

\section{CLINICAL SUMMARY}

A 22-year-old man with palliated hypoplastic left heart syndrome was referred for routine cardiac magnetic resonance evaluation. He underwent a Fontan operation at the age of 5 years and a TCPC with an atrial Maze procedure at the age of 14 years.
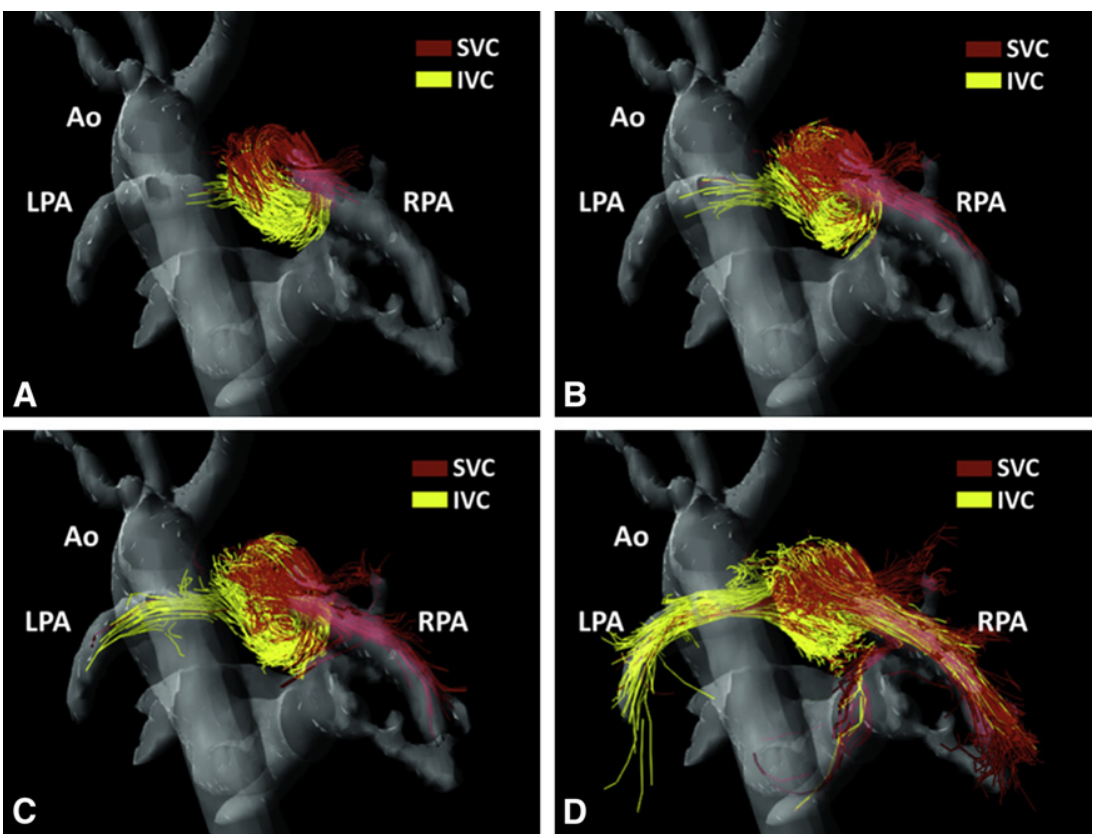

FIGURE 1. Particle traces emitted from the SVC (red) and IVC (yellow) into the pulmonary arteries along the cardiac cycle: $25 \%$ of cardiac cycle (A), $40 \%$ of cardiac cycle (B), $50 \%$ of cardiac cycle (C), and $80 \%$ of cardiac cycle (D). SVC, Superior vena cava; IVC, inferior vena cava; $R P A$, right pulmonary artery; $L P A$, left pulmonary artery; $A o$, aorta. 
By using a free-breathing 4-dimensional flow sequence, we quantified the caval flow distribution to the RPA and LPA with a new segmental approach. Imaging parameters were as follows: field of view, $200 \times 300 \mathrm{~mm}$; acquired voxel size, $2.5 \mathrm{~mm}^{3}$; reconstructed voxel size, $1.5 \times 1.5$ $\times 2.3 \mathrm{~mm}$; number of slices, 40; retrospective cardiac gating, number of signal averages $=1$; repetition time, $3.2 \mathrm{~ms}$; echo time, $1.9 \mathrm{~ms}$; flip angle, 5 degrees; sensitivity encoding $=2$; velocity encoding, $250 \mathrm{~m} / \mathrm{s}$; and temporal resolution, $49 \mathrm{~ms}$.

Four-dimensional flow sequence measures all 3 directional components of the velocities of blood flow relative to the 3 spatial dimensions along the course of the heart cy$\mathrm{cle}^{4}$ and is a reliable tool to quantify blood flow in patients with congenital heart disease. ${ }^{5}$

Particle traces derived from 4-dimensional flow data were used to quantify flow distribution. Particle traces are path-lines that imaginary particles follow over time according to the 4-dimensional flow velocity vector field. By defining a region of interest, particle traces can depict the spatial and temporal evolution of the bloodstream flow.

The proposed method consists of creating regions of interest in the SVC, IVC, RPA, and LPA. Particle traces were emitted from the region of interest located in the SVC and IVC. Thereafter, particles arriving to the RPA and LPA were counted, and percent of caval flow contribution to the RPA and LPA was calculated (GTFlow software; GyroTools LLC, Zurich, Switzerland).

Our results showed that $74.7 \%$ of the SVC blood flowed into the RPA and $25.3 \%$ flowed into the LPA. In contrast, $76.1 \%$ of the IVC blood flowed into the LPA and $23.9 \%$ flowed into the RPA (Figure 1). Although no PAVMs were found and the patient's oxygen saturations were normal, long-term follow-up studies may be required to evaluate the association of unbalanced caval flow distribution and the development of PAVMs.

\section{CONCLUSIONS}

This new method has the potential to (1) identify patients at risk of failing the Fontan procedure or TCPC with unbalanced right and left pulmonary flow distribution resulting from suboptimal surgical connections; (2) detect patients at risk of developing PAVMs, probably secondary to uneven IVC flow distribution; and (3) evaluate the results of different geometries of TCPC in vivo, such as the idealized geometry proposed by Dasi and colleagues. ${ }^{2}$

\section{References}

1. McElhinney DB, Marx GR, Marshall AC, Mayer JE, Del Nido PJ. Cavopulmonary pathway modification in patients with heterotaxy and newly diagnosed or persistent pulmonary arteriovenous malformations after a modified Fontan operation. $J$ Thorac Cardiovasc Surg. 2011;141:1362-70.

2. Dasi LP, Whitehead K, Pekkan K, de Zelicourt D, Sundareswaran K, Kanter K, et al. Pulmonary hepatic flow distribution in total cavopulmonary connections: extracardiac versus intracardiac. J Thorac Cardiovasc Surg. 2011;141:207-14.

3. Fogel MA, Weinberg PM, Rychik J, Hubbard A, Jacobs M, Spray TL, et al. Caval contribution to flow in the branch pulmonary arteries of Fontan patients with a novel application of magnetic resonance presaturation pulse. Circulation. 1999;99:1215-21.

4. Markl M, Kilner PJ, Ebbers T. Comprehensive 4D velocity mapping of the heart and great vessels by cardiovascular magnetic resonance. J Cardiovasc Magn Reson. 2011;13:7.

5. Nordmeyer S, Riesenkampff E, Crelier G, Khasheei A, Schnackenburg B, Berger F, et al. Flow-sensitive four-dimensional cine magnetic resonance imaging for offline blood flow quantification in multiple vessels: a validation study. J Magn Reson Imaging. 2010;32:677-83. 